\title{
Multilocular tuberculous cyst of thymus gland
}

\author{
Thomas Stephen, MS, ${ }^{a}$ Roy Thankachen, MCh, ${ }^{\mathrm{a}}$ Bhawna Parihar, MCh, ${ }^{\mathrm{a}}$ Sheila Nair, MD, ${ }^{\mathrm{b}}$ and \\ Vinayak Shukla, MCh, DNB, ${ }^{a}$ Vellore, India
}

A

lthough tuberculosis (TB) is widely prevalent in India, its involvement of the thymus is still a rare entity. We present here an asymptomatic patient incidentally found to have an anterior mediastinal mass, which on surgery turned out to be multilocular tuberculous cyst of the thymus. We describe the radiological and pathological findings and review of literature on TB of the thymus.

\section{Clinical Summary}

A 25-year-old man underwent a preemployment checkup and on chest skiagram was found to have a well-defined opacity in the region of the left hilum. A contrast-enhanced computed tomography (CT) scan of the chest revealed an anterior mediastinal mass of variegated consistency with solid and cystic areas measuring approximately $10.5 \times 6 \times 2.5 \mathrm{~cm}$ (Figure 1). There was a loss of plane with the pericardium and the arch of aorta with extension laterally to the upper lobe of the left lung. There was no associated pericardial effusion. The lung parenchyma appeared normal. $\beta$-human chorionic gonadotropin and alpha-fetoprotein markers as well as thyroid function tests were within normal limits. A CT-guided fine needle aspiration cytology/biopsy was not done because solid areas of the tumor were inaccessible.

Based on the preoperative investigations a differential diagnosis of thymic tumor, lymphoma, and tuberculous lymphadenitis was considered. Sternotomy and excision biopsy were planned. At surgery we found a large cystic mass occupying the left lobe of the thymus extending from the innominate vein to the apex of the heart. Laterally the mass was adherent to the upper lobe of the left lung. The pericardial cavity was opened and there were a few solid nodes occupying the area between the arch and the pulmonary artery adherent to the undersurface of the aorta. A frozen section of this mass was reported as TB. The entire cystic mass with the left lobe of thymus was then excised. The left pleura was opened and debulking of the adherent nodes was done to the extent possible.

Grossly the specimen measured $11 \times 6 \times 3 \mathrm{~cm}$. On section, it revealed a multiloculated cyst with cyst wall measuring $0.1 \mathrm{~cm}$ in

\footnotetext{
From the Departments of Thoracic and Cardiovascular Surgery and $\mathrm{Pa}$ thology, ${ }^{\mathrm{b}}$ Christian Medical College, Vellore, India.

Received for publication July 11, 2003; accepted for publication Aug 11, 2003.

Address for reprints: Dr Vinayak Shukla, Associate Professor, Department of Thoracic and Cardiovascular Surgery, Christian Medical College \& Hospital, Vellore 632 004, India (E-mail: shukla58@hotmail.com).

J Thorac Cardiovasc Surg 2003;126:2093-4

Copyright $\odot 2003$ by The American Association for Thoracic Surgery

$0022-5223 / 2003 \$ 30.00+0$

doi:10.1016/j.jtcvs.2003.08.008
}

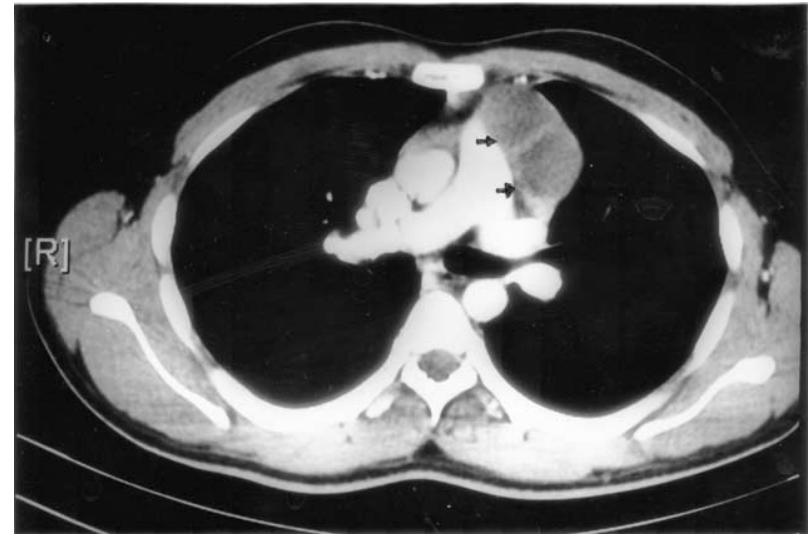

Figure 1. CT scan at the level of the pulmonary outflow tract showing multilocular thymic cyst in the anterior mediastinum (arrows).

diameter. It contained turbid and blood-stained fluid with small warty projections scattered over the inner lining of the cyst. Cut section of the lymph nodes revealed small foci of caseation. Histologically lymph nodes and thymic tissue showed chronic inflammation and multiple granulomas composed of epithelioid histiocytes and Langhans-type giant cells (Figure 2). Central caseous necrosis was present in the lymph nodes. There were no acid-fast bacilli on Ziehl-Neelsen stain. Subsequently the thymic tissue culture was positive for mycobacterium TB. His sputum smear neither showed acid-fast bacilli nor grew Mycobacterium tuberculosis organism on culture.

Postoperatively the patient was started on oral antituberculous treatment and recovered well.

\section{Discussion}

Tuberculosis of the thymus is an extremely rare condition, and in earlier reports it has been suggested to represent remnants of post-primary localized mediastinal lymphadenitis. ${ }^{1}$ To date only 3 cases have been reported, of which only 1 was culture-positive. ${ }^{1-3}$

A diagnostic approach for primary anterior mediastinal tumors has been evaluated in earlier studies. ${ }^{4}$ Accurate diagnosis is imperative in deciding upon surgical or nonsurgical therapy. Although CT scan is useful in assessing the extent of the lesion there are as yet no specific described CT findings in thymic TB. ${ }^{1}$ The role of needle aspiration biopsy is also controversial as many of the mass lesions of the anterior mediastinum have solid and cystic components and obtaining a representative tissue sample is difficult. In our patient, as well as in an earlier reported case, ${ }^{1}$ the mass appeared to have solid and cystic components on CT findings. This 


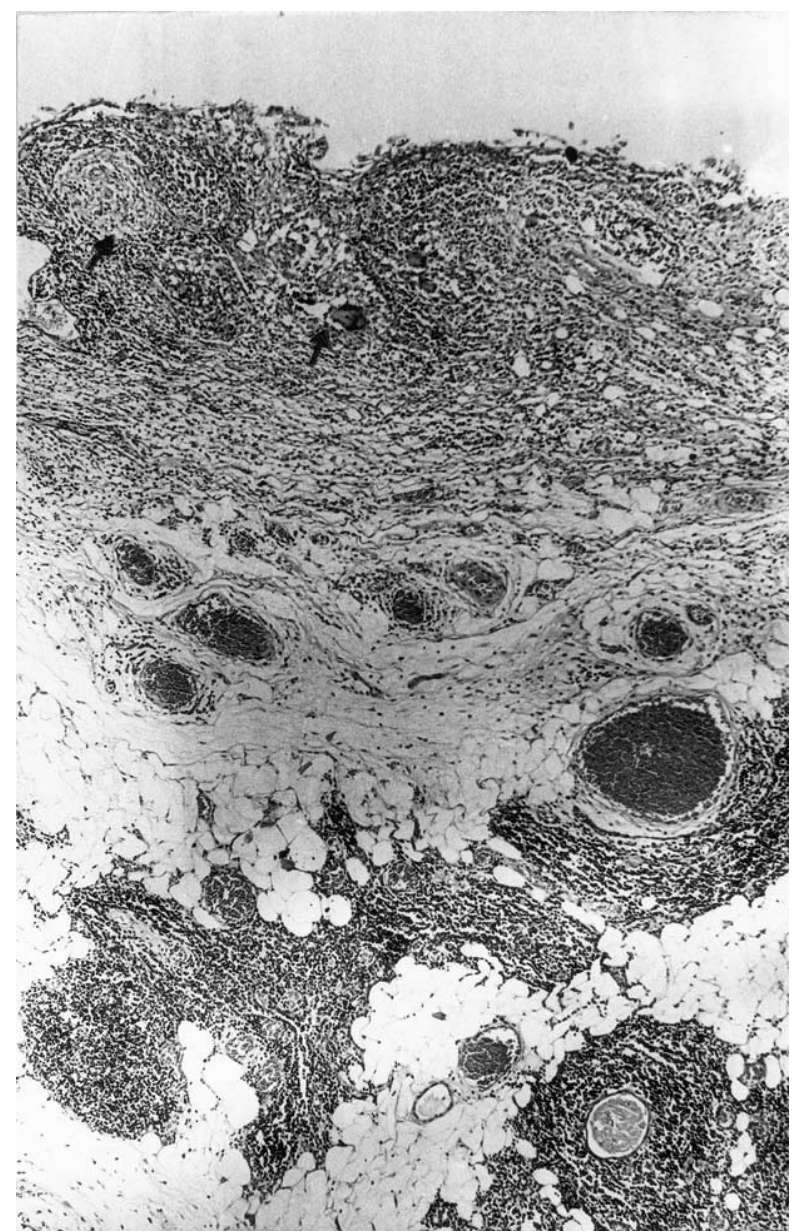

Figure 2. Low-power view of the thymic tissue (bottom) and epithelioid granulomas lining the cystic spaces (top; arrows). is a nonspecific feature considering that other lesions such as thymomas and germ cell tumors may present similar CT findings. There may be a role for mediastinoscopy/thoracoscopy in such cases where a preoperative diagnosis cannot be established although we were unable to do the same. ${ }^{4}$ However, exploratory thoracotomy has been recommended for all mediastinal tumors of undetermined etiology. 5

In conclusion, we present a case of anterior mediastinal mass incidentally picked up in an asymptomatic patient; the mass was found to be thymic TB at the time of surgery. This case demonstrates the diagnostic dilemma that can occur in patients with an anterior mediastinal mass and the need for an open biopsy for accurate histopathological diagnosis. In retrospect a high index of suspicion is needed to establish diagnosis nonsurgically.

We thank Mr Elumalai Ravikumar for help in the preparation of the manuscript.

\section{References}

1. Fitz Gerald JM, Mayo JR, Miller RR, Jamieson WRE, Baumgartner F. Tuberculosis of the thymus. Chest. 1992;102:1604-5.

2. Silvola HJ, Lahdesmaki M. On tuberculosis of the thymus. Ann Chir Gynaecol Fenn. 1966;55:27-30.

3. Duprez A, Cordier R, Schmitz P. Tuberculoma of the thymus. First case of surgical excision. J Thorac Cardiovasc Surg. 1962;44:11520.

4. Fradet G, Evans KG, Nelems B, Miller RR, Miller NL. Primary anterior mediastinal tumors: an investigational algorithm. Can J Surg. 1989;32: $139-42$.

5. Karlson KW, Timmes JJ. Granulomata of the mediastinum surgically treated and followed up to 9 years. J Thorac Surg. 1958;35: $617-27$. 